\title{
Responsabilidad Social Universitaria: Perspectiva y aportes desde una universidad confesional*
}

\author{
University Social Responsibility: perspective and contributions from a confessional university. \\ Responsabilidade Social Universitária: Perspectiva e as contribuições como Universidade Confessionais
}

DOI: https://doi.org/10.21803/pensam.v17121-1.275

\author{
Mallerlyn Ivaret Rodríguez de Rodríguez \\ https://orcid.org/0000-0001-6110-408X \\ Carlos William Marín Gallego \\ https://orcid.org/0000-0003-3454-4463
}

\section{¿Cómo citar este artículo?}

Rodríguez, M. \& Marín, G. (2018). Responsabilidad Social Universitaria: Perspectiva y aportes desde una universidad confesional. Pensamiento Americano, 11(22), 184-205. DOI: https://doi.org/10.21803/pensam.v17i21-1.275

\section{Resumen}

La Responsabilidad Social Universitaria (RSU) supone dos vertientes: una orientada a responder las demandas de sus públicos; y otra, dirigida a la trascendencia del actuar socialmente responsable a través de la formación de individuos. El propósito de esta investigación es describir la RSU en la Corporación Universitaria Adventista, a fin de mostrar la perspectiva y aportes como institución confesional hacia un modelo de gestión de la Responsabilidad Social Universitaria. Posee un enfoque mixto, y los instrumentos derivan del modelo de Vallaeys, De la Cruz y Sasia (2009). Los hallazgos muestran que muchos componentes de la RSU están implícitos en el accionar de la Corporación Universitaria Adventista, aunque no exista intencionadamente un modelo para gestionar la Responsabilidad Social Universitaria, tener los principios bíblicos como pilares de la gestión organizacional y de la formación, genera impactos positivos en sus diferentes públicos, resaltando la trascendencia de la formación integral que ofrece su proyecto educativo.

Palabras Clave: : Responsabilidad social universitaria, RSU, Formación integral, Universidades Confesionales, Educación adventista

\begin{abstract}
University Social Responsibility (USR) involves two aspects: one aimed at responding to the demands of its audiences; and another, aimed at the importance of acting socially responsible through the formation of individuals. The purpose of this research is to describe the USR in the Adventist University Corporation, to show the perspective and contributions as a confessional institution towards a management model of University Social Responsibility. It has a mixed approach, and the instruments derive from the Vallaeys, De la Cruz and Sasia model (2009). The findings show that many components of the USR are implicit in the actions of the Adventist University Corporation, although there is no intentionally a model for managing University Social Responsibility, having biblical principles as pillars of organizational management and training, generates positive impacts in its different audiences, highlighting the importance of the comprehensive training offered by your educational project.
\end{abstract}

Key words: University Social Responsibility, USR, Integral Formation, Confessional Universities, Adventist education.

* Artículo producto del Proyecto de Investigación “Situación actual de la Responsabilidad Social en la Corporación Universitaria Adventista. Hacia un modelo de gestión de la Responsabilidad Social Universitaria" 


\title{
Resumo
}

\begin{abstract}
A Responsabilidade Social Universitária (RSU) supõe duas vertentes: uma orientada a responder às demandas de seus públicos; e outro, dirigido à transcendência do ato socialmente responsável pela formação dos indivíduos. O objetivo desta pesquisa é descrever o RSU na Corporação Universitária Adventista, a fim de mostrar a perspectiva e as contribuições como instituição confessional em direção a um modelo de gestão da Responsabilidade Social Universitária. Ele tem uma abordagem mista, e instrumentos derivado do modelo Vallaeys, De la Cruz e Sasia (2009). Os resultados mostram que muitos componentes do RSU estão implícitos nas ações da Corporação Universitária Adventista, embora não intencionalmente há um modelo para gerenciar a Responsabilidade Social da Universidade, tem princípios bíblicos como pilares de gestão organizacional e formação, gera impactos positivos em seus diferentes públicos, destacando a importância da educação integral oferecida por seu projeto educacional.
\end{abstract}

Palavras-chave: Responsabilidade Social Universitária, RSU, Educação Integral, Universidades Confessionais, Educação Adventista

\section{Perfil}

Investigadora docente en Corporación Universitaria Adventista. Medellín, Colombia. mirodriguez@unac.edu.co. Especialista en gerencia de proyectos para el desarrollo. Contadora Pública. mirodriguez@unac.edu.co.

\section{Perfil}

Administrador de empresas. Investigador docente en Corporación Universitaria Adventista.Medellín, Colombia. cmarin@unac.edu.co. Magister en Administración de Empresas de la Universidad de Montemorelos, México. Especialista en Gerencia de Organizaciones. cmarin@unac.edu.co
Mallerlyn Ivaret Rodríguez de Rodríguez

Magister en Gerencia Pública

Instituto de Estudios Superiores de

Administración (IESA).

\section{Carlos William Marín Gallego}

Magister en Administración de

Empresas de la Universidad de

Montemorelos, México. 


\section{Introducción}

La sociedad actual demanda un replanteamiento de la responsabilidad de todos los actores que en ella confluyen. Es allí donde las instituciones educativas juegan un papel preponderante, por ser entes formadores de individuos para la vida en sociedad y principales generadores de conocimiento para la transformación. De acuerdo con Greenlaf citado en (Marin \& Páez, 2014) si el papel fundamental de las organizaciones es ser instituciones servidoras, lo cual se logra con un enfoque de servicio hacia sus públicos, cuánto más las universidades, a quienes se les ha confiado la tarea de formar a los agentes de cambio.

Por ende, se hace necesario que las instituciones de educación superior estén conscientes de su responsabilidad social y de la trascendencia de sus acciones, que sean capaces de contribuir al desarrollo sostenible, no sólo con su actuación como organización, sino con el legado que dejan en la generación de conocimiento y en la formación de personas aptas para aplicar esos conocimientos en pro del bien común.

En ese sentido, la responsabilidad social universitaria (en adelante RSU) cumple una doble función: una está orientada a la responsabilidad que debe asumir la institución como organización de la sociedad, donde debe responder a las demandas de sus diferentes públicos, tanto internos como externos; y otra, está dirigida a la trascendencia del actuar socialmente responsable que se transmite a través de la formación de individuos éticos, conscientes, participantes y promotores de los procesos de transformación social en favor del bienestar colectivo.

Las instituciones de educación superior deben formar a los estudiantes como ciudada- nos "dotados de principios éticos, comprometidos con la construcción de la paz, la defensa de los derechos humanos y los valores de la democracia". (Conferencia Mundial sobre la Educación Superior, 2009, p.2). Así mismo, se señala la importancia de una gestión de calidad pertinente a las necesidades del entorno, a través de la implantación de sistemas y el fomento de una cultura de calidad en las instituciones de educación superior (Conferencia Mundial sobre la Educación Superior, 2009).

Desde la perspectiva social, el planteamiento anterior muestra la demanda latente de personas éticas y socialmente responsables. Lo cual no es contrario al propósito misional de muchas universidades confesionales, las cuales según Gregorutti (2012) deben aportar "sabiduría", mencionando este término en relación a que ya no es suficiente con que los profesionales tengan conocimiento, sino que se requiere de personas íntegras con altas dosis de sabiduría a fin de evitar el efecto contraproducente de la posesión de conocimientos.

El autor menciona que muchas de las crisis sociales están relacionadas con crisis morales, basta con ver los casos de corrupción tanto empresariales como nacionales, los cuales han sido ocasionados por personas entrenadas y capaces, pero sin escrúpulos (Gregorutti, 2012). Como bien dijo Simón Bolívar "El talento sin probidad, es un azote".

Interpretando las ideas plasmadas por Gregorutti (2012), se puede decir que las universidades que se dedican además del entrenamiento profesional, a la formación en valores para la vida, tienen una gran responsabilidad.

Es allí donde cobra importancia visualizar la RSU desde una óptica espiritual.

En el contexto de la filosofía adventista, la

Pensamiento Americano Vol. 11 (22) • 2018 • Julio-Diciembre • Corporación Universitaria Americana • Barranquilla, Colombia • ISSN: $2027-2448$. 
crisis social es una crisis profética en la cual particularmente los adventistas y sus instituciones de educación superior, deben jugar un rol determinante en cuanto a aunar esfuerzos para mejorar y optimizar los servicios de gestión social universitaria. Se trata de asumir el liderazgo espiritual y académico de la gestión social, que la misma pueda ser de alto impacto hacia el prójimo.

Desde esa perspectiva, la apropiación del concepto de Responsabilidad Social en los adventistas representa la aplicación práctica de principios bíblicos y cristianos sustentados en el amor a Dios y al prójimo. Entendiendo como prójimo todos los públicos a los cuales impacta la organización.

Particularmente, en la Corporación Universitaria Adventista (en adelante UNAC) son muchas las iniciativas sociales y de servicio que se llevan a cabo, las cuales son plausibles porque reflejan la mejor disposición de la comunidad unacense a prestar un servicio desinteresado al prójimo, sin embargo, no es menos cierto que muchas veces resulta un tanto complejo medir el impacto que éstas dejan en todos sus públicos.

Las exigencias de la sociedad, por una parte, pero, sobre todo, el compromiso espiritual de dejar el mejor legado a la humanidad demandan ir un paso al frente y sistematizar todas esas iniciativas genuinas, donde se implemente de manera integral la responsabilidad social en cada una de las áreas de la gestión universitaria, con prácticas concretas perfectamente medibles e incluso replicables en otras instituciones de educación superior.

Por lo expuesto anteriormente, el presente documento con un enfoque mixto y mediante los instrumentos de recolección derivados del modelo de Vallaeys et al. (2009) tiene como objetivo describir la Responsabilidad
Social en la Corporación Universitaria Adventista (UNAC) como universidad confesional, con la finalidad de generar aportes hacia un modelo de gestión que permita sistematizar los procesos y medir la efectividad en el cumplimiento de estos. Modelo que se sustenta principalmente en dos enfoques: Uno, dirigido a la responsabilidad que la universidad tiene como organización propiamente dicha. Y otro, como ente formador de individuos socialmente responsables. Ambos en el marco de los principios bíblicos y cristianos, reconociendo a Dios como el creador, redentor y sustentador del Universo, fuente de todo conocimiento y sabiduría.

Esta investigación está fundamentada en principios bíblicos y cristianos de amor y servicio al prójimo, en la teoría de la responsabilidad social de las organizaciones desarrollada por diferentes referentes mundiales (ONU, Instituto Ethos, ISO 26000, Forética, entre otros) y en los postulados de Responsabilidad Universitaria de Vallaeys et al. (2009). Desde la perspectiva institucional, es coherente con el modelo pedagógico, el proyecto educativo institucional y las creencias cristianas que profesan las institucionales confesionales adventistas.

Se pretende que esta investigación conduzca a la comunidad universitaria a un proceso reflexivo sobre cómo se están llevando a cabo los aspectos claves relacionados al cumplimiento de la RSU en una universidad confesional en el ejercicio de su contribución misional a las demandas de la sociedad y de todos los públicos con los cuales interactúa, así como en la trascendencia en la generación de conocimiento para la transformación de la sociedad a través de la formación de individuos íntegros dotados de sabiduría.

Este documento muestra una breve reseña de la teoría de la responsabilidad social de las organizaciones a nivel general, y más 
específicamente el rol de la educación y la RSU, así como sus distintos enfoques. También, se presenta una contextualización del modelo educativo de la Corporación Universitaria Adventista (UNAC), y se enfatiza en la teoría de RSU de acuerdo a los postulados de Vallaeys et al. (2009), la cual sienta las bases para la metodología empleada en la presente investigación, que se caracterizó por un enfoque mixto, ya que se recogió información de tipo cualitativo y cuantitativo, empleando los instrumentos desarrollados por Vallaeys et al. (2009) para realizar el diagnóstico de RSU.

Diagnóstico que arrojó como resultado a la participación social como el área mejor valorada y la Gestión Social del conocimiento como el área menos valorada. Con esto se concluye que, aunque no exista en la Corporación Universitaria Adventista (UNAC) un modelo de gestión de la RSU, los diferentes públicos mostraron percepciones positivas en todas sus dimensiones, resaltando como valor agregado la creencia y prácticas sustentadas en principios bíblico-cristianos que permean la gestión organizacional y la formación integral de los estudiantes, elemento distintivo de esta universidad confesional.

\section{La responsabilidad social de las organiza- ciones.}

El término responsabilidad social ha cobrado auge en los últimos tiempos, sin embargo, resulta paradójico que no exista consenso a la hora de plantear una definición, en tal sentido, cada organización la define de acuerdo con sus paradigmas, sus valores y sus intereses.

A continuación, se muestran algunas de las definiciones propuestas por destacados referentes mundiales:

Es la responsabilidad de una organización ante los impactos que sus decisiones y ac- tividades (productos, servicios y procesos) ocasionan en la sociedad y en el medio ambiente, a través de un comportamiento transparente y ético que: contribuya al desarrollo sostenible, la salud y el bienestar de la sociedad; tome en consideración las expectativas de sus grupos de interés; cumpla con la legislación aplicable y es coherente con la normativa internacional; esté integrada en toda la organización y se lleve a la práctica en sus relaciones (ISO 26000, 2008).

De una manera muy explícita la ISO 26000 (2008) define la responsabilidad social por los impactos que genera en la sociedad y el medio ambiente. Otro aspecto interesante de esta definición es que hace referencia a la transparencia y la ética como elementos fundamentales de la responsabilidad social, adicionando la consideración con las expectativas que se crean en los diferentes grupos de interés.

Otra definición se encuentra en el Instituto Ethos (ONG brasilera que promueve la RSE):

Es una forma de gestión definida por la relación ética y transparente de la empresa con todos los públicos con los cuales se relaciona y por el establecimiento de metas empresariales compatibles con el desarrollo sustentable de la sociedad, preservando los recursos ambientales y culturales para las generaciones futuras, respetando la diversidad y promoviendo la reducción de las desigualdades sociales (Ethos, 2008).

La definición anterior propuesta por Ethos, al igual que la norma ISO 26000, destaca la importancia de la ética y la transparencia frente a los grupos de interés, así como la contribución al desarrollo sostenible.

Por su parte, Forética (Red de empresas y

Pensamiento Americano Vol. 11 (22) • 2018 • Julio-Diciembre · Corporación Universitaria Americana · Barranquilla, Colombia • ISSN: $2027-2448$. 
profesionales de la RSE), la define como:

Integración voluntaria (superando el cumplimiento estricto de las obligaciones legales vigentes) en el gobierno, gestión, estrategia, políticas y procedimientos de las organizaciones, de las preocupaciones sociales, laborales, medioambientales, y de respeto a los derechos humanos que surgen de la relación y el diálogo transparentes con sus grupos de interés, responsabilizándose así de las consecuencias y los impactos que se derivan de sus acciones (Forética, 2008).

En pocas palabras la definición anterior señala que todo lo que la empresa hace en pro del medio ambiente y/o de la sociedad que supera lo contemplado en la legislación existente es considerado responsabilidad social. Por supuesto, en esta definición también se señala la importancia de la transparencia con los grupos de interés y de la gestión de los impactos que la organización genera en el entorno.

En consecuencia, de las definiciones anteriores se desprende que Responsabilidad Social es:

- Gestión de impactos.

- Promover y garantizar desarrollo sustentable.

- Responder a las expectativas y demandas de los grupos de interés internos y externos.

- Es más que únicamente cumplir la ley.

- Es comportamiento transparente y ético.

\section{El rol de la educación y la RSU}

En 1960 aparece un movimiento intelectual en Suramérica haciendo énfasis en que las universidades no pueden continuar al margen de los problemas sociales, preocupación que avanzó progresivamente hasta ser tema en discusión de la Conferencia Mundial de Educación Superior de 1998 (Rodríguez, 2012, p.22).

Sin lugar a duda, la educación ha jugado siempre un papel fundamental en el desarrollo de las sociedades ha sido el mecanismo por excelencia del traslado del saber de generación en generación. No obstante, este saber adquiere verdadera utilidad cuando está orientado a la solución de los problemas reales de la sociedad. No en vano la UNESCO reconoce la vital importancia de la educación básica para el progreso social (UNESCO, 1998).

Es de hacer destacar la doble vía que tiene la educación, donde aporta a la sociedad a la vez que reconoce el entorno como insumo para el conocimiento.

Según el enfoque de Amartya Sen (1998) el bienestar humano está determinado por el aumento de las capacidades, entendidas éstas como la posibilidad de hacer más cosas, "la habilidad para llevar el tipo de vida que consideran valiosa e incrementar sus posibilidades reales de elección" (p.69).

En este aspecto, la educación es de vital importancia para el mejoramiento de la calidad de vida.

Por su parte, Vallaeys et al. (2009) señala que el paradigma de la RSU supera el enfoque de la proyección social y extensión universitaria, por tanto, propone una reflexión integral de la universidad como institución académica que influye en el entorno social. En ese sentido, la RSU desde una perspectiva integral demanda una articulación de los diferentes frentes institucionales en un proyecto ético y de desarrollo social para la transmisión de saberes responsables y la formación de individuos responsables.

Considérese entonces, las universidades 
como agentes de desarrollo, entendiendo el desarrollo como "una ampliación de la libertad humana" (Sen, 1999, p.2), por tanto tienen el compromiso de realizar las reformas correspondientes en sus programas de estudio y de practicar una gestión ética, a fin de tener personas más libres para elegir el tipo de vida que desean vivir, ésta libertad en el contexto cristiano viene dada principalmente por el conocimiento de la verdad expresada en la palabra de Dios. "Si vosotros permaneciereis en mi palabra, seréis verdaderamente mis discípulos y conoceréis la verdad, y la verdad os hará libres" (Jn 8:31-32 Reina Valera).

\section{Enfoques de la RSU}

En el contexto de la RSU es importante precisar los principales aportes que realizan las diferentes teorías económicas, entre ellas se señalan la teoría de la agencia, la teoría de los stakeholders, la teoría de la legitimidad, la teoría institucional, y la teoría basada en recursos y capacidades (Larrán \& Andrades, 2015).

La teoría de agencia centra la atención en la autonomía y la rendición de cuentas (Larrán \& Andrades, 2015).

La teoría de los stakeholders hace referencia a las demandas y expectativas de los diferentes públicos, así como la participación de estos en los procesos de evaluación de la gestión universitaria. (Gaete, 2009).

Partiendo de la teoría de la legitimidad, las universidades incorporan la RSU como un mecanismo para mejorar la imagen, reputación corporativa y legitimación social (Larrán \& Andrades, 2013).

Desde el enfoque de la teoría institucional, se menciona que las universidades implementan la RSU en respuesta a las presiones sociales ejercidas por el entorno (Larrán \& Andra- des, 2015).

Por su parte, la teoría de recursos y capacidades muestra la RSU como un elemento diferenciador capaz de generar ventajas competitivas (Larrán \& Andrades, 2015).

De todas las teorías anteriormente mencionadas se pueden destacar aspectos claves que bien pueden complementarse para la implementación de un modelo que permita gestionar la RSU. La teoría de la legitimidad y la teoría institucional apuntan a la dimensión externa de la RSU, la primera, para proyectar una imagen o reputación corporativa y la segunda, en reacción a demandas o presiones sociales. De igual forma, la teoría de recursos y capacidades postula que la RSU trae en consecuencia ventajas competitivas, orientándose en ese sentido también hacia la proyección externa. Sin embargo, es importante mencionar que, si bien es cierto que la gestión de impactos hacia los públicos externos es fundamental, no se puede dejar de lado el ejercicio de la Responsabilidad a lo interno de las universidades.

Es por esto, que la teoría de los stakeholders coloca los cimientos para la implementación integral de la Responsabilidad Social Universitaria.

Por su parte, las universidades confesionales pueden aportar una visión de la responsabilidad social de forma integral, incorporando elementos adicionales, ya que el relacionamiento con los diferentes públicos es una consecuencia del relacionamiento con Dios.

La idea de la responsabilidad hacia los demás es un mandato divino (Kliskberg, 2004) de acuerdo con el principio bíblico que reza "Amarás a tu prójimo como a ti mismo" (Mateo. 22:39, Versión Nueva Reina Valera, 2000), se encomienda la responsabilidad ética de velar por los demás (Kliskberg, 2004) y este 
"velar" puede ser entendido como la internalización de que en el accionar universitario se deben considerar los impactos internos y externos de la gestión, pero no por evitar presiones sociales como plantea la teoría institucional sino por la concepción moral y ética de que es un principio de vida fundamentado en la biblia (Gregorutti, 2012).

Un objetivo manifiesto de las instituciones educativas cristianas es generar transformación y cambios para las personas y para la sociedad en general (Knight, 2002). Pero, en muchos casos no existen elementos sustanciales que las distingan de las instituciones no confesionales, en las primeras se incorpora la religión como un anexo a la praxis educativa convencional, pero sin mayor trascendencia para la vida de las personas y para el entorno en el cual operan (Knight, 2002).

Desde esa perspectiva, es un reto para las universidades confesionales llevar a la práctica su compromiso misional fomentando y practicando la justicia, la solidaridad y el desarrollo responsable (Robles, 2017).

Las universidades confesionales del siglo XXI, evidencian como elementos distintivos: Una cosmovisión que incorpora la dimensión espiritual del ser, un propósito de desarrollo del carácter a través de una relación con Dios, un currículo que incorpora en su literatura libros sagrados como fuente de sabiduría, un cuerpo docente profesional con valores y vidas ejemplares, un alumnado que reconoce la dimensión espiritual, y una interacción con la cultura que impacta todas las dimensiones del quehacer humano (Gregorutti, 2012). Todas estas características propician más que la generación de conocimiento, el fomento de la sabiduría (Gregorutti, 2012).

De acuerdo con los preceptos bíblicos, la verdadera sabiduría es la veneración y respeto a
Dios, por tanto, la educación de valor es aqueIla orientada a presentar esta veneración y respeto sobre cualquier otra enseñanza. Si bien es cierto que los conocimientos académicos son importantes, superior es el conocimiento de Dios y sus principios. Es importante la formación para la vida actual, más importante aún la preparación para la vida eterna.

Tal como plantea White (2009):

Es necesario que tenga una mayor amplitud y un fin más elevado. La verdadera educación significa más que la prosecución de un determinado curso de estudio. Significa más que una preparación para la vida actual. Abarca todo el ser, y todo el período de la existencia accesible al hombre. Es el desarrollo armonioso de las facultades físicas, mentales y espirituales. Prepara al estudiante para el gozo de servir en este mundo, y para un gozo superior proporcionado por un servicio más amplio en el mundo venidero (p. 13).

Desde esa perspectiva, existen tres propósitos de la educación cristiana, los cuales se detallan en la figura 1.

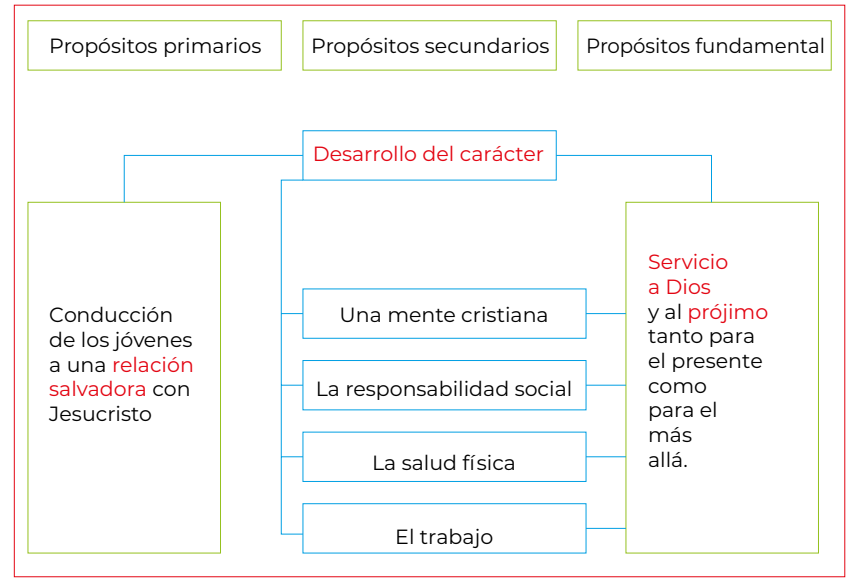

Propósitos de la educación crisitana para maestros. George R. Knight. 2002

Figura 1. Propósitos de la educación cristiana

Fuente: Knight (2002). 
La figura 1 muestra que la filosofía de la educación adventista resalta la función que tiene en dos ámbitos complementarios, no excluyentes uno de otro. Uno obedece a la formación para la vida en sociedad, y otro a la preparación para la vida eterna. Su elemento diferenciador es que ofrece además del desarrollo de habilidades y competencias para el mejoramiento personal, el desarrollo de dones para glorificar a Dios, que se materializan en el servicio hacia los demás y hacia la sociedad.

Particularmente, temas que actualmente se identifican como manifestaciones de la responsabilidad social, tales como la conservación y cuidado del medio ambiente, el respeto por la dignidad humana y la cordialidad y solidaridad en las relaciones interpersonales, desde la óptica adventista están estrechamente relacionados con el servicio al Dios creador y el servicio al prójimo (Iglesia Adventista del Septimo Día, 2011).

En ese sentido, los adventistas defienden la idea de que se les ha concedido la responsabilidad de ser "Mayordomos", con este símil se destaca el reconocimiento de que nada es posesión propia, sino que todo pertenece a Dios, y, por tanto, su función es administrar de la mejor manera todo lo que se les ha confiado.

Sobre este fundamento se condena toda forma de discriminación, se aboga por un trato justo hacia los más desfavorecidos, se apoyan los objetivos de Desarrollo de las Naciones Unidas, se participa y se patrocinan programas para erradicar la pobreza y el hambre, se practica el cuidado de la naturaleza, se exhorta a llevar un estilo de vida que se abstenga del tabaco, bebidas alcohólicas y el consumo de drogas, se actúa conjuntamente con las autoridades gubernamentales en proyectos locales, nacionales y/o mundiales a fin de es- tablecer una justicia más duradera, y, en lo que respecta al lugar de trabajo, se promueve un ambiente de servicio y respeto mutuo, se defiende la libertad de conciencia, se procuran salarios y condiciones de trabajo justos, entre otros (Iglesia Adventista del Septimo Día, 2011).

\section{Prácticas educacionales: enfoque cristiano de la educación.}

En su intención de impulsar la formación cristiana, este tipo de educación incluye prácticas que pretenden que "el estudiante adopte o profundice las creencias cristianas, los valores y actitudes así como la disposición para actuar de manera cristiana" (León, 2012, p.90). Específicamente, desde la perspectiva educativa adventista, se considera la educación como redentora (Arana et al., 2015) como afirma Taylor (2012), "La educación cristiana busca restaurar la imagen de Dios." (p.98). Esto implica que la educación debe asumirse como un proceso intencional que conlleve a este resultado deseado, por lo cual las prácticas educacionales deben fundamentarse en la cosmovisión cristiana bíblica.

George Knight, reconocido filósofo y educador adventista, asevera que "el cristianismo es una religión de transformación y cambio tanto para el individuo como para las sociedades" (Knight, 2002, p.172), por ello propone una relación entre la filosofía con la práctica educacional que asegure coherencia e intencionalidad. En ese sentido, propone unos determinantes filosóficos (ver gráfica 1) que están relacionados con la naturaleza de la realidad (creencias metafísicas), la esencia de la verdad (creencias epistemológicas) y una base para formar valores (creencias axiológicas), que condicionan las prácticas educacionales (Knight, 2002).

También los elementos del entorno, como las

Pensamiento Americano Vol. 11 (22) • 2018 • Julio-Diciembre · Corporación Universitaria Americana · Barranquilla, Colombia • ISSN: $2027-2448$. 


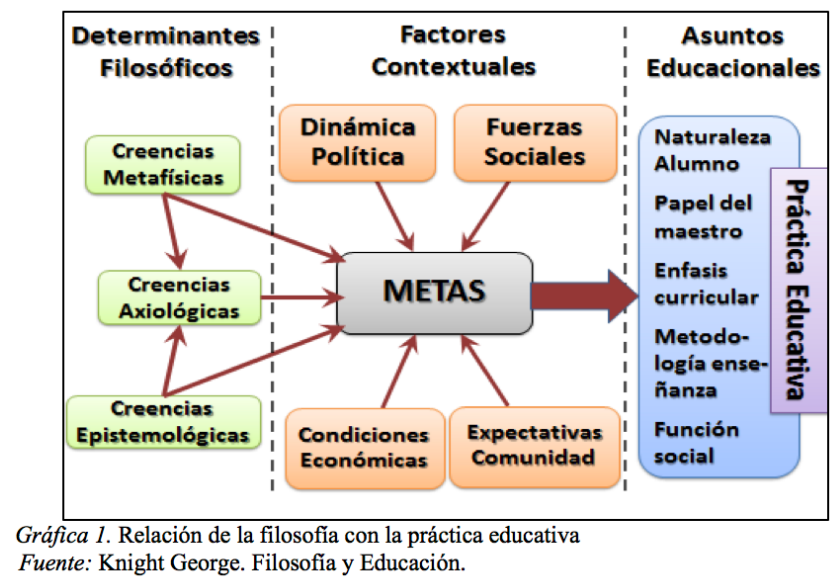

fuerzas políticas, las condiciones económicas, las necesidades del mercado y las concepciones sociales, impactan las prácticas educativas concluyendo que lo que se requiere en las instituciones cristianas es un programa permanente de análisis, evaluación y mejoramiento de las prácticas educacionales en conformidad con las creencias filosóficas básicas del cristianismo (Knight, 2002). Así se sientan las bases para construir un modelo educativo que responda a estos esenciales filosóficos y misionales.

\section{Modelo educativo de la Corporación Univer- sitaria Adventista (UNAC)}

La Corporación Universitaria Adventista cuenta con cinco facultades: Educación, Teología, Salud, Ciencias Administrativas y Contables e Ingenierías. Donde se ofrecen como programas de formación profesional: Licenciatura en español e inglés, Licenciatura en Educación Infantil, Licenciatura en Educación Religiosa (acreditado en alta calidad), Licenciatura en Música (acreditado en alta calidad), Licenciatura en Matemáticas, Enfermería, Tecnología en Atención Prehospitalaria, Administración de Empresas, Contaduría Pública, Tecnología en Mercadeo y Ventas, Ingeniería de Sistemas.

También, se ofrecen programas de especia- lización profesional: Especialización en Docencia, Especialización en Gestión Tributaria y Especialización en Gerencia de las Organizaciones.

La Corporación Universitaria Adventista (UNAC) declara su misión: "Propiciar una relación transformadora con Dios en el educando por medio de la formación integral en las diferentes disciplinas del conocimiento, preparando profesionales competentes, éticamente responsables, con un espíritu de servicio altruista a Dios y a sus semejantes, dentro del marco de la cosmovisión bíblico-cristiana que sustenta la Iglesia Adventista del Séptimo Día" (Corporación Universitaria Adventista, 2016, p.16).

Como visión señala que "será una comunidad universitaria adventista con proyección internacional, reconocida por su alta calidad, su énfasis en la formación integral, la cultura investigativa y la excelencia en el servicio, que forma profesionales con valores cristianos, comprometidos como agentes de cambio con las necesidades de la sociedad y su preparación para la eternidad" (Corporación Universitaria Adventista, 2016, p.16).

Esa formación integral, de acuerdo con la propuesta pedagógica está orientada a desarrollar todas las dimensiones del ser: intelectual, espiritual, física y social.

La dimensión intelectual, se concibe como "la capacidad individual o colectiva de realizar procesos de razonamiento para interpretar, argumentar y evaluar la realidad, e intervenir y proponer acciones de mejoramiento como una forma de garantizar el desarrollo y el progreso de la sociedad" (Corporación Universitaria Adventista, 2016, p.22).

La dimensión espiritual, se concibe como "la capacidad del individuo para construir su 
proyecto de vida en armonía con la creencia de que existe un propósito divino para cada ser humano, cuya aceptación permite desarrollar más plenamente todas sus potencialidades, para su propio progreso y el servicio a sus semejantes" (Corporación Universitaria Adventista, 2016, p.22).

La dimensión física, "se define como la capacidad del individuo para entender que su cuerpo es creación de Dios y que como tal debe conocer y desarrollar hábitos que le motiven a mantener óptimas condiciones de salud que le permitan llevar a cabo las actividades que se proponen" (Corporación Universitaria Adventista, 2016, p.23).

La dimensión social, se refiere a "la necesidad de construir relaciones sociales asertivas que incentiven a la persona a desenvolverse adecuadamente en los ambientes en que interacciona, elevando su calidad de vida a nivel individual y colectivo" (Corporación Universitaria Adventista, 2016, p.23).

La dimensión intelectual se desarrolla a través del uso de metodologías que promuevan una reflexión crítica de contenidos actualizados, como resultado del proceso investigativo. La espiritual, a través de actividades que propician un ambiente de comunión con Dios, la integración de la fe y la enseñanza a lo largo del currículo, y a través del estilo de vida de docentes con valores cristianos. La física a través de la promoción de hábitos saludables que incluyen la alimentación, el descanso, el ejercicio y la recreación. La dimensión social, se desarrolla a través de la participación en diferentes actividades culturales, recreativas, deportivas y de servicio a la comunidad (Corporación Universitaria Adventista, 2016).

\section{Modelo de gestión de responsabilidad so- cial universitaria por Vallaeys et al. (2009)}

El modelo de Vallaeys et al. (2009) se basa en la gestión de los impactos que la institución universitaria genera en su entorno, los cuales se agrupan básicamente en cuatro categorías: organizacional, educativa, cognitiva y social. Con respecto a los impactos organizacionales, como cualquier organización, la universidad impacta en la vida de su personal (docentes y empleados administrativos), así como sus clientes (que en este caso particular son los estudiantes). Así mismo, con su operación impacta al medio ambiente.

En cuanto a los impactos sociales, la institución tiene una responsabilidad en cuanto al desarrollo social y a la solución de problemas de la vida cotidiana. La universidad tiene un alcance social, como figura que puede fomentar el progreso, desarrollar capital social, y propiciar el relacionamiento de los estudiantes con la realidad social, hacer accesible el conocimiento a todos, etc. (Vallaeys et al., 2009).

En el caso específico de los impactos educativos, la universidad es responsable por la formación de los jóvenes y profesionales, sus valores, la percepción del mundo y su forma de comportarse en él (Vallaeys et al., 2009). En otro orden, la universidad tiene impactos cognitivos, "influye en la definición de lo que socialmente se llama verdad, ciencia, racionalidad, legitimidad, utilidad, enseñanza, etc." (Vallaeys et al., 2009, p. 9). Tiene la facultad para propiciar la apropiación social del conocimiento, e influir en la definición y selección de los problemas que marcan el rumbo científico de las sociedades (Vallaeys et al., 2009). Este modelo describe cuatro ejes para implementar la responsabilidad social en las instituciones universitarias, los cuales se presentan en la figura 2. 


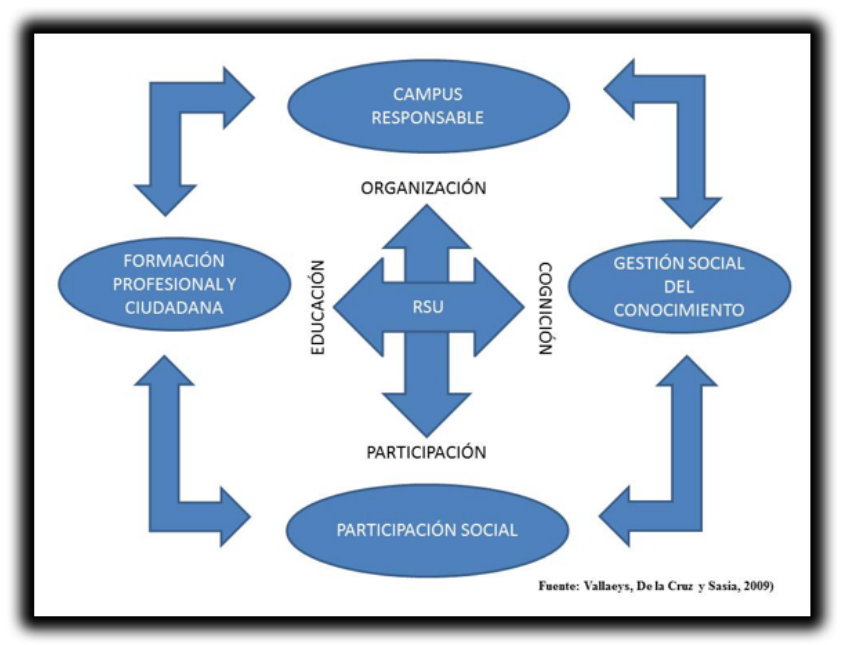

Figura 2. Ejes de la Responsabilidad Social Universitaria. Fuente: Vallaeys et al. 2009

El campus responsable, implica la gestión de la responsabilidad social de la universidad como organización, por tanto, cobran especial interés los procedimientos institucionales, el clima organizacional, las políticas de gestión humana, los procesos democráticos internos y la preservación ambiental (Vallaeys et al., 2009).

La formación profesional y ciudadana, se refiere a la gestión de la formación académica (la malla curricular, la metodología, la propuesta didáctica y la temática), la cual debe ser complementada con la formación de competencias ciudadanas, éticas, morales y de responsabilidad social, así como la pertinencia con los problemas reales y actuales de la sociedad. Para lograr este objetivo, el modelo propone que se tome en cuenta a los grupos de interés al momento de diseñar los programas de estudio, ya que son los empleadores, los gremios profesionales y otros actores tanto internos como externos quienes se ven beneficiados o perjudicados por la formación que reciben los estudiantes (Valleys et al., 2009).

La gestión social del conocimiento apunta a que la actividad científica que se produce dentro de la universidad esté a tono con las exigencias del ámbito local y nacional, es decir con las agendas de desarrollo social y proyectos del sector público, así como la difusión de estos conocimientos generados para los públicos interesados, que el conocimiento no quede en las bibliotecas, sino que por el contrario tenga verdadera utilidad práctica en la sociedad (Valleys et al., 2009).

La participación social es fundamental para la responsabilidad social universitaria, puesto que se orienta al diseño y ejecución de proyectos con otros actores para el establecimiento de redes (capital social) para el aprendizaje mutuo y el desarrollo social. Este eje propone el trabajo en equipo entre personal universitario y no universitario en pro de proyectos sociales, de manera que la acción colectiva garantice un constante aprendizaje y al mismo tiempo contribuya a la solución de problemas sociales específicos (Valleys et al., 2009).

Estos cuatro ejes se retroalimentan permanentemente para la mejora continua de la responsabilidad social, garantizando pertinencia social en el aspecto organizacional y en el aspecto académico (Valleys et al., 2009).

\section{Método}

La presente investigación tiene un enfoque mixto ya que recoge información tanto cuantitativa como cualitativa. Como aprecia Pereira (2011) "los diseños mixtos permiten la obtención de una mejor evidencia y comprensión de los fenómenos y, por ello, facilitan el fortalecimiento de los conocimientos teóricos y prácticos" (p.19).

El diseño se considera de modelo mixto, confirmatorio, de tipo simultáneo, ya que usa datos cuantitativos y cualitativos, con análisis cuantitativos y cualitativos (Pereira, 2011, p.20). 
La población estudiada está representada por los públicos en los que tiene impacto la actuación de la Corporación Universitaria Adventista (UNAC), tales se dividen en: Grupos internos y grupos externos. La tabla 1 muestra la clasificación de ambos grupos, así como los instrumentos utilizados con cada uno de ellos.

Tabla 1.

Públicos en los que impacta la Corporación Universitaria Adventista (UNAC)

\begin{tabular}{|c|c|c|c|c|}
\hline & Tipo de público & Población & Muestra & Instrumento \\
\hline \multirow{6}{*}{$\begin{array}{l}\text { Grupos de } \\
\text { interés } \\
\text { internos }\end{array}$} & Estudiantes & 1231 & 293 & Encuesta \\
\hline & Docentes & 156 & 95 & Encuesta \\
\hline & Investigadores & 22 & 20 & Encuesta \\
\hline & $\begin{array}{l}\text { Coordinadores de } \\
\text { proyección social }\end{array}$ & 5 & 5 & Encuesta \\
\hline & $\begin{array}{l}\text { Personal no } \\
\text { profesoral }\end{array}$ & 57 & 46 & Encuesta \\
\hline & $\begin{array}{c}\text { Autoridades } \\
\text { (Rector, } \\
\text { Vicerrectores, } \\
\text { Directora de } \\
\text { Desarrollo } \\
\text { Humano, Director } \\
\text { de } \\
\text { Investigaciones, } \\
\text { Coordinadores de } \\
\text { Proyección } \\
\text { Social) }\end{array}$ & & 6 & Entrevistas \\
\hline \multirow{7}{*}{$\begin{array}{l}\text { Grupos de } \\
\text { interés } \\
\text { externos }\end{array}$} & Egresados & & 3 & Entrevista \\
\hline & $\begin{array}{l}\text { Empleadores de } \\
\text { egresados }\end{array}$ & & 2 & Entrevista \\
\hline & $\begin{array}{c}\text { Familiares del } \\
\text { personal }\end{array}$ & & 2 & Entrevista \\
\hline & Proveedores & & 2 & Entrevista \\
\hline & $\begin{array}{l}\text { Comunidad } \\
\text { vecina }\end{array}$ & & 1 & Entrevista \\
\hline & $\begin{array}{l}\text { Comunidad } \\
\text { adventista }\end{array}$ & & 2 & Entrevista \\
\hline & $\begin{array}{l}\text { Comunidad } \\
\text { beneficiaria de las } \\
\text { acciones de } \\
\text { proyección social }\end{array}$ & & 2 & Entrevista \\
\hline
\end{tabular}

Fuente: Los autores. 


\section{Resultados}

Tomando toda la información de las encuestas en conjunto, se tiene una media para la variable Campus Responsable de 4.2, Formación Ciudadana y Profesional 4.2, Gestión Social del Conocimiento 3.5 y Participación Social 4.4. (Ver tabla 2).

En un intento por diagnosticar la situación general de la Corporación Universitaria Adventista (UNAC) en cuanto a RSU, la figura 3 muestra cada eje de la RSU y la puntuación obtenida de las encuestas, siendo la escala máxima seis (6). Se aprecia que todos los ejes presentan valores cercanos a cuatro (4). Siendo el área menos valorada Gestión Social del conocimiento y el área mejor valorada Participación Social. Mientras que Campus Responsable y Formación ciudadana presentan el mismo promedio.

Tómese en cuenta que de acuerdo al modelo sugerido por Vallaeys et al. (2009) para este

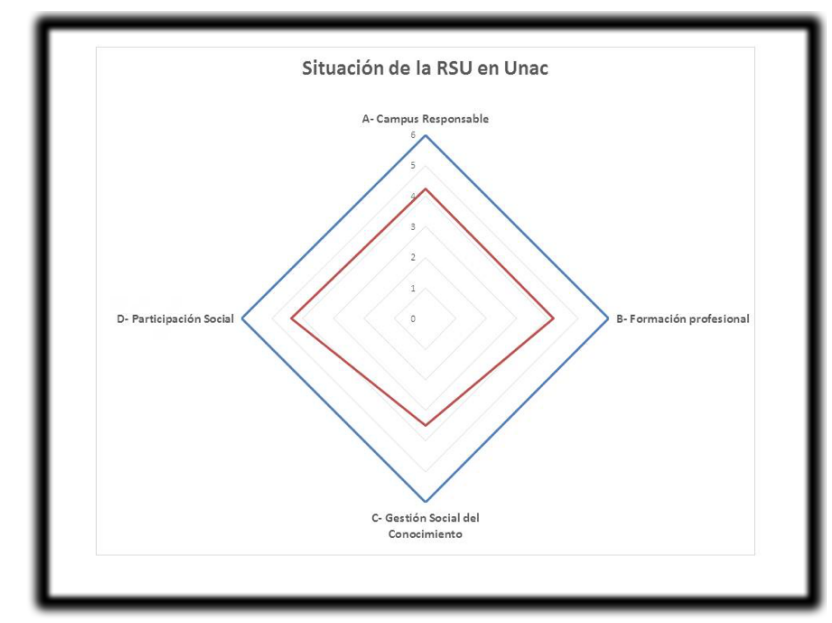

Figura 3. Diagnóstico cuantitativo de la Responsabilidad Social en la Corporación Universitaria Adventista Fuente: Los autores.

diagnóstico, el eje Gestión Social del Conocimiento, fue evaluado sólo por un grupo de participantes (en este caso de investigadores), mientras que en el resto de los ejes participó más de un grupo.

A continuación, las tablas 3, 4, 5 y 6 muestran de forma resumida los aspectos más resaltantes del diagnóstico cuantitativo y cualita-

Tabla 2.

Promedio general ejes de la RSU.

\begin{tabular}{|c|c|c|c|c|c|c|c|}
\hline Ejes RSU & Escala & Promedio & $\begin{array}{c}\text { Estu- } \\
\text { diantes }\end{array}$ & Docentes & $\begin{array}{c}\text { Personal } \\
\text { no } \\
\text { profesoral }\end{array}$ & $\begin{array}{l}\text { Investi- } \\
\text { gadores }\end{array}$ & $\begin{array}{c}\text { Coordi- } \\
\text { nadores de } \\
\text { proyecció } \\
\text { n social }\end{array}$ \\
\hline $\begin{array}{c}\text { A-Campus } \\
\text { Responsable }\end{array}$ & 6 & 4,2 & 4,17 & 4,4 & 4,11 & & \\
\hline B- Formación & & & & & & & \\
\hline $\begin{array}{l}\text { Ciudadana y } \\
\text { Profesional }\end{array}$ & 6 & 4,2 & 4,23 & 4,16 & & & \\
\hline C- Gestión & & & & & & & \\
\hline $\begin{array}{c}\text { Social del } \\
\text { conocimiento }\end{array}$ & 6 & 3,5 & & & & 3,5 & \\
\hline D- & & & & & & & \\
\hline $\begin{array}{c}\text { Participación } \\
\text { Social }\end{array}$ & 6 & 4,4 & 3,99 & & & & 4,78 \\
\hline
\end{tabular}

Fuente: Los Autores. 
Tabla 3.

Resultados del diagnóstico en la dimensión Campus Responsable

\section{Campus Responsable}

\begin{tabular}{ll}
\hline \multirow{3}{*}{ Estudiantes } & - Existe un trato de respeto y colaboración entre profesores y estudiantes. \\
& - Las relaciones interpersonales en general son de respeto y cordialidad. \\
& - No hay discriminación por género, raza, nivel socioeconómico u orientación \\
& política o sexual. \\
& - Dentro de la universidad se promueve el trabajo en equipo y la solidaridad. \\
Docentes & - Existe un buen clima laboral. \\
& - Existe coherencia entre los principios que declara la universidad y lo que se \\
practica en el campus. & - Los docentes perciben que no existe discriminación en el acceso a la docencia, \\
& ni por género, religión, raza, orientación política o sexual.
\end{tabular}

Personal no - En la universidad se promueve el trabajo en equipo y la solidaridad.

Autoridades - La naturaleza de la institución y el requerimiento de los entes acreditadores favorecen el respeto a derechos humanos, equidad de género y no discriminación. - La universidad es confesional pero abierta a todos los públicos sin distingo de raza, partidos políticos, condiciones socioeconómicas, siempre en el marco de la filosofía institucional.

- Existe un compromiso con la inclusión, y la responsabilidad social, en primer lugar porque así lo demanda el estado, y en segundo lugar, porque representa la esencia de la institución.

- La responsabilidad social se concibe con un sentido de visión, imitando a Jesús, quien con su ejemplo mostró que el servicio es la acción de interesarse por los demás con una generosidad y espíritu de abnegación extraordinario.

- Por filosofía propia de las instituciones adventistas, éstas funcionan en un entorno ambiental que favorece los procesos enseñanza-aprendizaje, a la vez que permite a los estudiantes ver una expresión del amor de Dios a través de la naturaleza, considerado el segundo libro de Dios, luego de la Biblia.

- Las instituciones adventistas fundamentan su quehacer en la cosmovisión bíblico-cristiana, se interesan en el cuidado del medio ambiente y tienen que estimular en los estudiantes el cuidado del mismo.

- En la institución existe un departamento encargado de recoger, clasificar y reciclar la basura, en el marco de la política de responsabilidad con el medio ambiente.

- La institución es $100 \%$ libre de humo, mientras otras universidades luchan con este flagelo, el ser libre $100 \%$ de humo es un gran aporte que la UNAC realiza al medio ambiente.

\section{Familiares del personal}

- Se otorgan importantes beneficios laborales para el trabajador y su familia, auxilios para estudio de los trabajadores y sus hijos, auxilios de vivienda.

- Muchos profesores y personal administrativo viven dentro del campus universitario junto con su familia.

Proveedores La universidad llena las expectativas de los proveedores en cuanto a trato respetuoso y transparencia en los procesos de contratación.

Fuente: Los autores 
Tabla 4.

Resultados del diagnóstico en la dimensión Formación profesional y ciudadana.

\section{Formación Profesional y ciudadana}

- La universidad brinda una formación ética y ciudadana que ayuda a los

Estudiantes estudiantes a ser personas socialmente responsables.

- La formación es integral, humana y profesional, y no sólo especializada.

- Los diversos cursos que se dictan están actualizados y responden a necesidades sociales del entorno.

- La universidad brinda a los estudiantes una formación ética y ciudadana que los ayuda a ser personas socialmente responsables.

Docentes

- Se vinculan los contenidos temáticos enseñados con los problemas sociales y ambientales de la actualidad.

- En los cursos los estudiantes tienen que hacer actividades que impactan positivamente en el entorno social.

Autoridades - Desde la filosofía institucional se promueve la formación integral en las diferentes disciplinas del conocimiento, esa formación integral incluye el respeto a derechos humanos, equidad, e inclusión a nivel transversal de todo el currículo.

- Dentro de la estrategia de formación integral, se cuenta con el programa de labor educativa, que es un programa incluyente orientado a estudiantes de bajos recursos donde a la vez que se forman en un programa profesional, se vinculan laboralmente a las diversas áreas de apoyo de la institución, recibiendo no sólo la experiencia laboral sino recursos que les permiten cubrir los costos educativos y servicios conexos, participando también de todas las actividades espirituales y de fortalecimiento integral en todas las dimensiones del ser que promueve la institución.

Egresados - Los valores espirituales y la sensibilidad social hacia las necesidades de los demás constituyen el elemento diferenciador de los egresados.

Empleadores - Los egresados se distinguen por su ética profesional y formación espiritual.

Comunidad - La Unac llena las expectativas en cuanto a la formación profesional y espiritual de los estudiantes.

Adventista

- La misión de la universidad es coherente con la misión de los adventistas.

- La universidad tiene como valor agregado la enseñanza acerca del amor de Jesús.

Fuente: Los autores 
tivo, que describen la RSU en la Corporación Universitaria Adventista (UNAC), así como los aportes que ésta realiza a la RSU desde su perspectiva confesional.

\section{Discusión}

Los resultados de la investigación muestran que la dimensión Participación Social fue el área mejor valorada con un promedio general de 4,4. La dimensión Gestión Social del conocimiento fue el área menor valorada con un promedio general de 3,5; y las dimensiones Campus Responsable y Formación Profesional y Ciudadana presentaron un promedio general de 4,2 siendo 6 el valor máximo de la escala.

Específicamente, la percepción de los públicos está orientada a que en la dimensión Campus Responsable las fortalezas más destacadas están asociadas al trato respetuoso, buen clima laboral, y no discriminación, lo cual está fundamentado en la cosmovisión bíblica que Gregorutti (2012) menciona como uno de los aportes o elementos distintivos de las universidades confesionales, lo cual se vive no sólo en el ámbito formativo sino en la praxis diaria en el recinto universitario, y que de acuerdo a las teorías de R.S. constituyen un elemento fundamental a lo interno de las organizaciones.

Así mismo se observan buenas relaciones con todos los públicos, y desde la perspectiva institucional se resalta el valor que el medio ambiente y la convivencia institucional tienen a la luz del cumplimiento de principios bíblico-cristianos.

En la dimensión Formación Profesional, se destaca la formación ética y ciudadana, considerado como un elemento diferenciador coherente con la filosofía y el proyecto educativo institucional, que de acuerdo con Gregorutti (2012) se expresa en la integración de la ciencia y la fe en el currículo educativo.

En la dimensión Gestión Social del Conocimiento, se encontró que los estudiantes

Tabla 5 .

Resultados del diagnóstico en la dimensión Gestión Social del Conocimiento

\section{Gestión social del conocimiento}

\section{Investigadores}

Autoridades
- Los estudiantes de pregrado deben obligatoriamente practicar la nvestigación en varios cursos de su formación.

- Los investigadores opinan que los proyectos y programas de investigación incorporan sistemáticamente a los estudiantes.

- Se considera transcendente que la investigación que se desarrolle contribuya a la solución de problemáticas tanto propias de la institución como de la iglesia y de la comunidad en la cual se participa, se busca que la investigación científica sea aplicada.

- Se cuenta con una línea de investigación en Desarrollo Social, donde se promueve la actividad investigativa orientada a la solución de problemas sociales y al bienestar integral y sustentable de la humanidad.

Fuente: Los autores 
Tabla 6.

Resultados del diagnóstico en la dimensión Participación Social

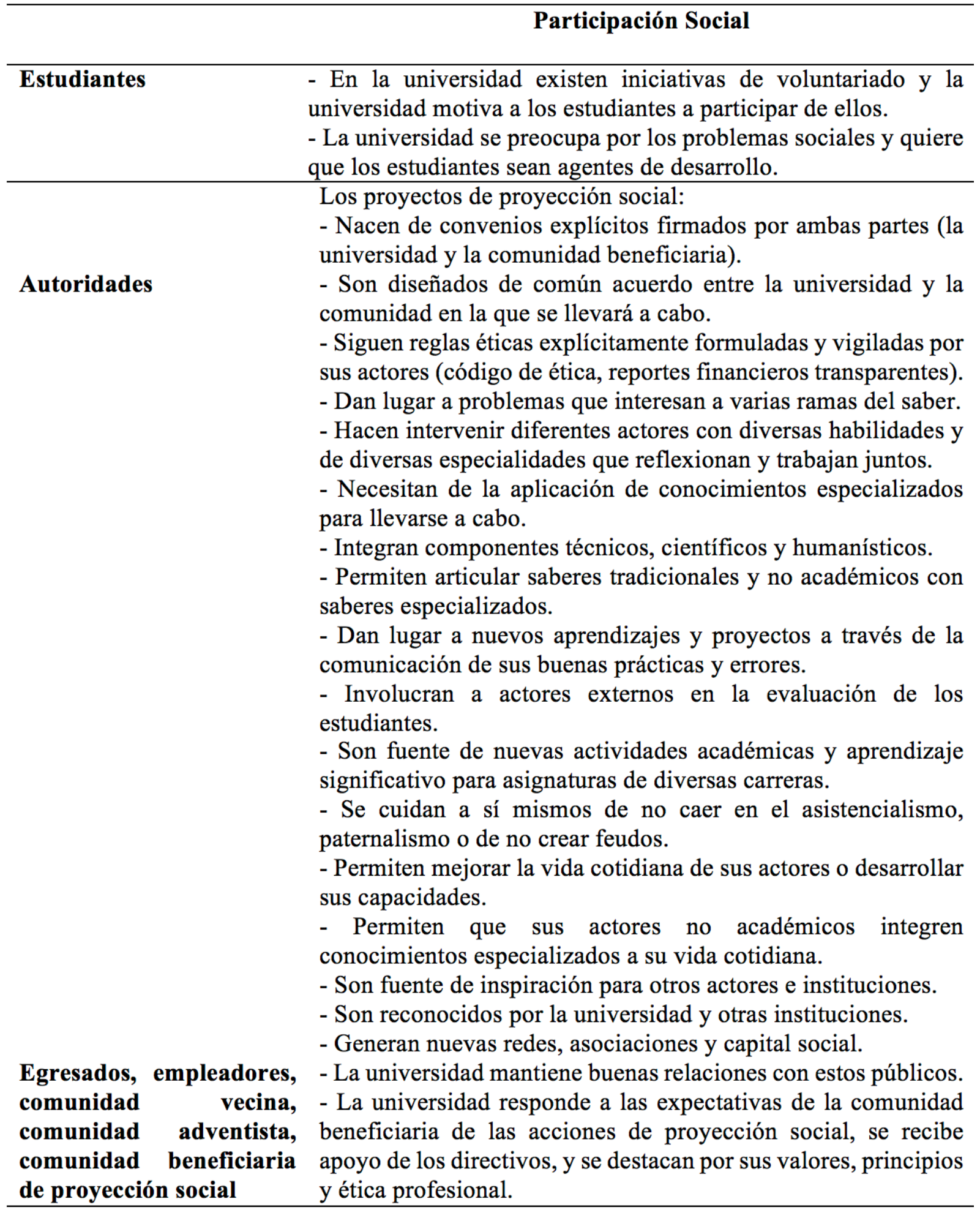

Fuente: Los autores. 
participan en proyectos de investigación en el curso de su formación, interactuando con la cultura para promover una visión proactiva del paradigma que sustenta (Gregorutti, 2012). No obstante, se detectaron algunas oportunidades de mejora en relación con el desarrollo de investigaciones para atender necesidades de las comunidades locales, lo cual representa un indicador de la RSU de acuerdo al modelo de valleys et al. (2009).

En la dimensión Participación Social se encontró que la universidad promueve el voluntariado y la incorporación de los estudiantes en actividades que benefician a la comunidad, integrando el entrenamiento profesional y científico con la construcción de valores (Gregorutti, 2012) asociados a la solidaridad y amor al prójimo. Se concibe el servicio como una consecuencia del amor a Dios más que una práctica altruista enfocada en la bondad de quien lo practica (Knight, 2002).

De acuerdo con las bases teóricas consultadas en este trabajo investigativo, la RSU se gestiona desde cuatro dimensiones: Campus Responsable, Formación Profesional y Ciudadana, Gestión del Conocimiento y Participación Social. Las cuales, desde la perspectiva de la institución confesional estudiada en esta investigación, se conciben como:

Campus Responsable: Hace referencia a la eficiente gestión de la organización y el cuidado de las personas que en ella conviven, entendiendo que el campus como recinto físico, y las personas como seres humanos son creación de Dios; y que, por tanto, existe una responsabilidad de origen divino de administrar el entorno y sus habitantes de manera beneficiosa para la sociedad.

Formación Profesional y ciudadana: Se refiere al desarrollo del carácter, es decir talentos, habilidades y virtudes para el servicio a Dios y a la sociedad. Formación orientada a la búsqueda de sabiduría, es decir la aplicación de los conocimientos para el bien común y con propósitos eternos.

Gestión del conocimiento: Se describe como la búsqueda de la verdad, con la convicción de que todo conocimiento proviene de Dios, por lo tanto, las investigaciones integran las teorías científicas con la fe, prevaleciendo los principios divinos expresados en la biblia.

Participación Social: Es la concreción de los principios y creencias cristianas, se refiere al servicio a los demás, siguiendo el modelo de Jesucristo, quien se caracterizó por atender las necesidades de los más desfavorecidos, y un amor abnegado, incondicional, altruista, sin esperar más retribución que la satisfacción del deber cumplido.

De manera que, la concepción de la RSU desde la perspectiva de la Corporación Universitaria Adventista se puede resumir en el ejercicio de la "Mayordomía Cristiana", la cual es conocida por los adventistas como la correcta administración de todo lo que está bajo su dominio, entiéndase medio ambiente, recursos financieros, tiempo, talentos y hasta la vida misma, tanto propia como de las personas que les rodean.

\section{Conclusiones y Recomendaciones}

De acuerdo a los resultados obtenidos en la investigación, se puede concluir que a pesar de que no se ha establecido un modelo de Responsabilidad Social Universitaria en la Corporación Universitaria Adventista (UNAC), en líneas generales el diagnóstico arrojó percepciones positivas por parte de los diferentes públicos, tanto internos como externos, lo cual se sustenta en la filosofía institucional, donde más allá de las exigencias de la sociedad el accionar está altamente influenciado

Pensamiento Americano Vol. 11 (22) • 2018 • Julio-Diciembre • Corporación Universitaria Americana • Barranquilla, Colombia • ISSN: $2027-2448$. 
por una conducta que obedece a valores supremos de amor al prójimo, respeto a la dignidad humana, y vocación de servicio, así como un profundo compromiso de dejar el mejor legado a la humanidad.

En otro orden, siendo que la responsabilidad social es un proceso permanente de mejora continua, que busca la perfectibilidad de la institución a través del autoaprendizaje constante, se recomienda sistematizar las iniciativas de RSU a través de la implementación de un modelo de gestión.

El modelo de gestión de la RSU de Valleys et al. (2009), comprende las fases que se muestran en la figura 4. La institución confesional tiene gran parte del camino recorrido, ya que se ha dado el primer paso representado por el compromiso, el cual es intrínseco a su misión y filosofía institucional. La presente investigación constituye el segundo paso (autodiagnóstico), el cual reflejó en gran parte el tercer paso (cumplimiento), se recomienda comenzar a transitar el cuarto paso, correspondiente a la rendición de cuentas.

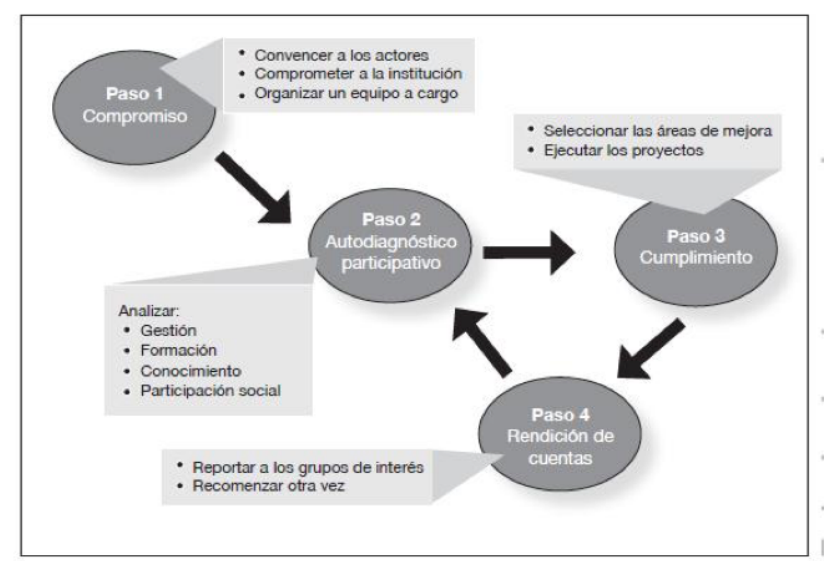

Figura 4: Fases para la implementación de un modelo de gestión de la Responsabilidad Social Universitaria.

Fuente: Vallaeys et al. (2009).
Desde la perspectiva de la universidad confesional que sirvió de objeto de estudio en esta investigación, la RSU representa la concreción de la mayordomía cristiana, a través del cuidado del medio ambiente, los recursos, y el desarrollo de las personas en su dimensión mental, social, física y espiritual.

Su modelo educativo, se sustenta en el desarrollo mental, social, físico y espiritual de los estudiantes, de igual manera, la institución como "Ser" también se compone de estas dimensiones. Por tanto, la figura 5 representa gráficamente la interpretación del modelo de RSU para la Corporación Universitaria Adventista, el cual puede ser replicable en otras instituciones de naturaleza similar.

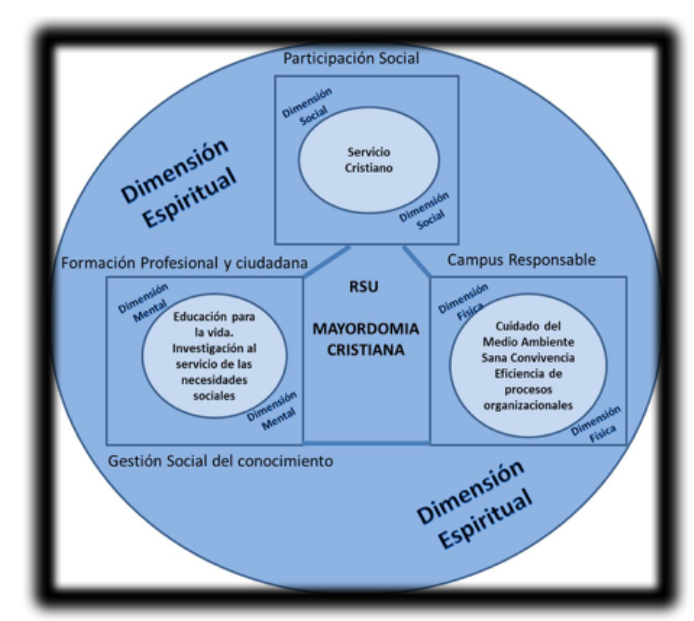

Figura 5: Modelo de RSU para la Corporación Universitaria Adventista Fuente: Los Autores. 


\section{Referencias}

Arana, W., Chaparro, D., Iglesias, E., Padilla, J., Pérez, G., Phérez, G., \& Sinza, D. (2015). Propuesta pedagógica Unac. Medellín, Colombia. Recuperado de: https:// www.unac.edu.co/wp-content/uploads/PDF/normatividad/10.Propuesta-Pedagogica-UNAC-2015. pdf

Conferencia Mundial sobre la Educación Superior. (2009). La nueva dinámica de la educación superior y la investigación para el cambio social y el desarrollo. Obtenido de http://www.unesco.org/ education/WCHE2009/comunicado_es.pdf

Corporación Universitaria Adventista. (2016). Proyecto Educativo Institucional.

Ethos. (2008). Preguntas frecuentes. Obtenido de http:// www.ethos.org.br/DesktopDefault.aspx?Tabl$\mathrm{D}=3344$ \&Alias=Ethos\&Lang=pt-BR.

Forética. (2008). Documentación Curso de Gestión de Responsabilidad Social Empresarial. Madrid.

Gaete, R. (2009). Participación de los stakeholders en la evaluación del comportamiento socialmente responsable de la gestión universitaria: perspectivas, obstáculos y propuestas. Comunicación presentada en el Congreso de AECA, Valladolid.

Gregorutti, G. (2012). La universidad confesional y los nuevos modelos de universidades: ¿Es posible mantener la identidad? Apunter Universitarios. Revista de investigación(1), 9-20.

Iglesia Adventista del Septimo Día. (2011). Declaraciones, orientaciones y otros documentos. Colombia: Gema editores, Asociación publicadora interamericana.

ISO 26000. (2008). Guía sobre responsabilidad social ISO 26000: borrador ISO 26000 WD4.2. Obtenido de http://isotc.iso.org/livelink/livelink/7490356/ NT041_v.2_traducci_n_WD4.2_a_aprobar_en_Santiago? func $=$ doc. Fetch\&nodeid $=7490356$
Kliskberg, B. (2004). El impacto de las religiones sobre la agenda social actual. Revista Valores en la sociedad industrial, 22(604), 62-71.

Knight, G. R. (2002). Filosofía y Educación: Una introducción en la perspectiva cristiana. Bogotá, Colombia: Asociación Publicadora Interamericana.

Larrán, M. \& Andrades, F. (2013). Frenos y aceleradores para la implantación de la responsabilidad social en las universidades españolas. Prisma Social Revista de Investigación Social, 10. 233-270.

Larrán, M. \& Andrades, F. J. (2015). Análisis de la Responsabilidad Social universitaria desde diferentes enfoques teóricos. Revista iberoamericana de educación superior, 91-107.

León, V. (2012). Importancia y efectividad de la filosofía educativa Adventista en Chile. Apuntes Universitarios. Revista de Investigación, 1, 87-126.

Marin, C. W. \& Páez, D. (2014). Aplicación del liderazgo seridor en las organizaciones. Sotavento MBA(23), 108-129.

Pereira. (2011). Los diseños de método mixto en la investigación en educación : Una experiencia concreta. Revista Electrónica Educare, XV(I), 15-29.

Pereira Pérez, Z. (2011). Los diseños de método mixto en la investigación en educación : Una experiencia concreta. Revista Electrónica Educare, XV(I), 15-29.

Robles, L. (2017). Propuesta metodológica de la Responsabilidad Social Universitaria para la Universidad Santo Tomás. Bogotá, Colombia.

Rodríguez, J. (2012). La Responsabilidad Social es inherente a la naturaleza y misión de la universidad. $L a$ Responsabilidad Social Universitaria en la educación a distancia. 13-37.

Sen, A. (1998). Human Capital and Human Capacity. Cuadernos de Economía, XVII (29), 67-72. 
Sen, A. (1999). Romper el ciclo de la pobreza: Invertir en la infancia. Conferencia Magistral, BID. Obtenido de http://www.iadb.org/sds/doc/SOC\%2D114S.pdf

Taylor, J. W. (2012). Posmodernidad y educación cristiana: Desafíos ideológicos contemporáneos. Enfoques, (2), 85-100. Retrieved from http://www.redalyc.org/pdf/259/25926198002.pdf

UNESCO. (1998). Declaración mundial sobre educación para todosy el marco de acción para satisfacer las necesidades básicas de aprendizaje. $7^{\circ}$ ed. Nueva York.

Vallaeys, F., De la Cruz, C., \& Sasia, P. (2009). Responsabilidad Social Universitaria. Manual de Primeros Pasos. México, D.F.: Mc Graw Hill.

White, E. G. (2009). La educación. Florida: Asociación Publicadora Interamericana. 